\title{
RESHAPING THE LEARNING EXPERIENCE THROUGH APPS: AFFORDANCES
}

\author{
$\underline{\text { Nigel Calder }}$ \\ University of Waikato \\ New Zealand
}

\author{
Carol Murphy \\ University of Waikato \\ New Zealand
}

This paper reports on how the affordances of the app Math Shake reshaped the learning experience - an aspect of a research project examining the ways mobile technologies are used in primaryschool mathematics. Students used different digital tools within the app to solve word problems, while the affordances, including multi-representation, dynamic and haptic, made the learning experience different from when using pencil-and-paper technology. However, while the affordances of the MT are important, the teacher's pedagogical approach was central in the learning.

\section{Introduction}

There has been a proliferation in the availability and use of mobile technologies (MT) over the last five years, including in educative settings. Their low instrumentation and ease of operation, coupled with the interaction being focused primarily on touch and sight, make using them intuitive for learners. Linked to this increase in MT is the growth in educational apps. Questions have been raised regarding the appropriateness of the content and pedagogical approaches of apps (e.g., Philip \& Garcia, 2015) but if MT are an inevitable and relatively enduring element of the evolving digital world, we need to consider their potential for learning. This paper reports on an aspect of a research project examining the ways iPad apps, as an example of MT, are used in primary-school mathematics. The project considers the pedagogy that might best facilitate the learning with students (ages 7-10) when engaging in mathematics through MT. In particular, the paper reports on the affordances of the app Math Shake and how they might be seen to reshape learning experiences in primary mathematics.

The use of digital technologies has the potential for introducing new ways of engaging with mathematical concepts and processes, and for re-envisaging aspects of mathematical education, along with alternative ways to facilitate understanding (e.g., Borba \& Villareal, 2005; Calder, 2011). Borba and Villarreal's (2005) perspective saw understanding emerging from the reconciliation of re-engagements of the collectives of learners, media, and environmental aspects with the mathematical phenomena. Borba and Villarreal contend that each engagement re-organises the mathematical thinking and initiates a fresh perspective that in turn transforms the nature of each subsequent engagement with the task. This iterative process of re-engaging with the task from each new perspective continues until some form of shared negotiated understanding occurs (Calder, 2011).

Meanwhile, Meyer (2015) suggested that MT offer a socio-material bricolage for learning. Drawing on Fenwick and Edwards' (2012) notion of socio-material approaches to learning, she envisaged complex systems where resources interact with knowledge distributed across people, communities and sites of practice. She used the term socio-material bricolage to describe the "ecological entanglement of material and social aspects of teaching and learning with technology” (Meyer, 


\section{Calder \& Murphy}

2015, p. 28). The notion of bricolage suggests that there is a mutually influential collective of tools and users affecting the dialogue, learning experience, and mathematical thinking, in particular and personalised ways. In an associated viewpoint, de Freitas and Sinclair (2014) identified fingerscreen-voice-five assemblages through which a child was involved in a rhythmic engagement when counting to five with the Touch Counts app. They contend that these assemblages were not static but fluent, and might manifest in varying constitutions and combinations.

\section{Affordances}

In relation to Gibson's (1977) notion of affordance as the complementarity of the learner and the environment and to Brown's (2005) acknowledgement of the potential relationship between the user and the artifact, the visual and dynamic affordances of MT may be seen to fashion the learning experience in distinctive ways, and so reposition students' engagement with mathematics.

One affordance frequently associated with digital environments is the notion of multiple representations. The ability to link and simultaneously interact with visual, symbolic, and numerical representations in a dynamic way has been acknowledged extensively in research (e.g., Ainsworth, Bibby \& Wood, 1998; Calder, 2011). Sacristán and Noss (2008) illustrated how the engagement of computational tasks in a carefully designed microworld might lead to different representational forms (such as visual, symbolic and numeric): a process that they called representational moderation. In a similar way, various studies involving dynamic geometry software, report that the dynamic, visual representations enhanced the understanding of functions (e.g., Falcade, Laborde \& Mariotti, 2007). This dynamic affordance, coupled with the propensity to give instant feedback to input, transforms the nature of the learning experience compared to pencil-and-paper technology.

Virtual manipulatives (VM) are frequently part of mathematical apps. They are described as interactive, web-based visual representations of dynamic objects (Moyer, Bolyard \& Spikell, 2002) that might afford opportunities for mathematical thinking. In Math Shake word problems are generated at various levels, and it provides a range of digital pedagogical tools (e.g., empty number lines, counters, ten frames), that students can select to help with their solutions. VM offer potential to extend the learning experiences with representations beyond those with pencil-and-paper medium (Arcavi \& Hadas, 2000). Reporting on a meta-study comparing the influence of VM to physical manipulatives and textbook instruction on the learning process, Moyer-Packenham and Westenskow (2013) indicated that while there was no significant statistical difference in achievement between the two approaches, there was a moderate averaged effect size when the effects were analysed by mathematical domains, grade levels, and study duration.

Moyer-Packenham and Westenskow (2013) identified the affordances of focused constraint, creative variation, simultaneous linking, efficient precision and motivation when students used apps in their mathematical learning. While these affordances interact, and appear to be mutually influential of each other, the Math Shake app appears to afford three of them in particular: focused restraint, where the app might focus students' attention on particular mathematical concepts or processes; creative variation, where the app might encourage creativity, hence evoking a range of student approaches and potential solutions; and simultaneous linking, where the app might link representations simultaneously and connect them to student activity (Moyer-Packenham \& Westenskow, 2013). In Math Shake a word problem is generated, and the students highlight the 
words associated with the appropriate mathematical process (suggesting focused constraint). They can select tools, such as an empty number line, to help with solving the problem (suggesting focused constraint and creative variation) while these representations can be linked to each other and also to the students' actions in the form of immediate feedback (suggesting simultaneous linking). The connections made between pictorial and symbolic representations, mediated through the actions executed on these representations, can emphasize the associated mathematical concepts and processes (Moyer-Packenham \& Westenskow, 2013). In a study examining the differences in learning effects of virtual and physical manipulatives, the importance of students' facility with representations and tools was confirmed (Moyer-Packenham et al., 2013). They also indicated that a lack of familiarity with different representations might negatively influence students' understanding.

As well as having affordances similar to other digital technologies, such as the opportunity to engage dynamically, the glass interface of an iPad presents a further affordance through touch. Student interaction is more directly responsive to input, enhancing the relatively high agency of the medium. There is direct interaction with the phenomena, rather than being mediated through a mouse or keyboard, making the iPad more suitable for young children than desktop computers (Sinclair \& Heyd-Metzuyanim, 2014). Apps might use this haptic affordance (e.g., with Multiplier, where within the task, the student drags out the visual area matrix associated with multiplication facts). This app also evokes multi-touch functionality, enabling students to make sense of individual effects of particular screen touches (Hegedus, 2013), and to create personal explanations of their thinking. This is similar to the simultaneous linking and creative variation that Moyer-Packenham and Westenskow (2013) identified.

Much of the discussion regarding the ways iPads and apps might transform the learning experience, is centred on the notion of student engagement (e.g., Attard, 2015) with students being actively enthralled and interested, often by the visual and interactive characteristics of the pedagogical medium (Carr, 2012). Others have indicated that affordances of digital technologies, together with the associated dialogue and social interaction, may lead to students exploring powerful ideas in mathematics, learning to pose problems, and create explanations of their own (Sandholtz, Ringstaff, \& Dwyer, 1997). They also reported improved high-level reasoning and problem solving linked to learners' investigations in digital environments. iPads and apps also foster experimentation (Calder \& Campbell, 2016), allowing space for students to explore. The apps affordances of interactivity and instantaneous feedback foster the learner's willingness to take cognitive risks with their learning (Calder \& Campbell, 2016). They allow students to model in a dynamic, reflective way. Others contend that MT can provide new forms of personal ownership (e.g., Meyer, 2015) that in turn supports learners' personal understanding and conceptual frames (Melhuish \& Falloon, 2010).

In a similar way to apps such as Explain Everything, Math Shake allows students to record individual or group presentations of mathematical processes, strategies and solutions. Further to this, Math Shake generates word problems at different levels that might be explored through the range of digital pedagogical tools students can select to assist with realizing their solutions. The screencasting feature of the app, and the simplicity with which it is enabled on an iPad, opens up other learning opportunities that would not be possible with approaches that use pencil-and-paper as pedagogical tools exclusively. Such an app introduces a further multi-representational affordance 


\section{Calder \& Murphy}

that can create an aural representation that students can listen to. In this paper we report on teachers' and students' perceptions of the learning opportunities afforded through the use of Math Shake, as an example of an app that uses screencasting, the digital recording of the computer screen, along with voice recording.

\section{Methodology}

The research used an interpretive methodology that relates to building knowledge and developing research capability through collaborative analysis and critical reflection of classroom practice and student learning. The research design is aligned with teacher and researcher co-inquiry whereby the university researchers and practicing teachers work as co-inquirers and co-learners (Hennessy, 2014), with an emphasis on collaborative knowledge building. Three teachers, all experienced with using MT in their programmes were involved in the study. One teacher taught a year-4 class using a BYOD approach, while the other two teachers team-taught in a year-5 \& 6 class. Data, obtained through different sources (focus group interviews, classroom observations, interviews with teachers, and blogs) were analysed using NVivo via a mainly inductive or grounded method to identify themes. Refinement of the identified themes occurred through joint critical reflection between teacher practitioners and academic researchers in research meetings. In this paper we present extracts of data from the teacher and student interviews concerning use of the Math Shake app. These responses are analysed in relation to the emerging themes of the project.

\section{Results}

\section{The teacher interviews:}

One teacher commented on the direct interface of the iPad screen, suggesting that the students were interacting more directly with the content of the mathematics. "Like a physical object that they're interacting with.” As well as the haptic affordance, this suggests focused restraint as the teacher perceived the app facilitating more direct interaction with mathematical content. The teacher further explained how apps involving screencasting for recording students' strategies were powerful agents in learning as the students were "creating something...explaining their own thinking, creating their own content, their own language.” This teacher comment points to the notion of creative variation affordance. The students are creating their own content and language, hence differentiating the experience and learning to some extent.

Another teacher noted how screencasting enabled less confident students to explain their thinking in a "nonthreatening environment" with "no teacher staring at them, no other kids waiting for them to hurry up.” “They're in a safe place where they can just record their thinking without any pressure.”

The teacher also saw benefits in assessing the students' thinking as the recording provided them with an understanding of "what's going on in the kid's head." Also in giving feedback to the students on their learning. The non-threatening environment for feedback seemed to resonate with the simultaneous linking affordance. Here the linking was between the student's action and the instantaneous, onscreen feedback.

\section{The Student interviews:}

Several students referred to the idea of drawing on the iPad screens or of tapping to select a tool and how this made their work "easier and tidier." This suggests the haptic affordance. Use of 
screencasting to record their solution strategies seemed a key feature for the students. The students talked of videoing themselves doing maths, and recording their working out. As one said, "It's just like making a movie for maths.” The opportunity to record their voices whilst writing and drawing seemed important as it was "hard to explain without writing down. You can write it down as well as explaining it while you're recording.” This student comment suggests that they are utilising the multi-representational affordance to articulate their explanation of the strategy that they are using. It also has elements of simultaneous linking, between the recording, writing and drawing features. The opportunity to pause and edit their recordings also appeared to be significant. "The cool thing is that you can actually pause it and then think about what you're going to do." Students also commented on the assurance that they had a correct solution, and hence had confidence in their strategies to start recording, "when you have your question and you’ve got your equation right.”

Some students commented how the feedback and opportunity to record their solutions had helped their learning in mathematics "We can write things down and answer questions to see if we are right or wrong." This comment also has elements of the linking between the student actions and the feedback resulting from the mathematical processes taking place within the digital learning environment. Others referred to the opportunity to use the different tools on the iPad and how these introduced them to new strategies: "I like learning new strategies; using a number line and place value." Some students noted specific instances of learning: "I learnt how to use the reversing strategy on the number line.” These student comments suggested focused constraint, with the app focusing the student's attention on particular mathematical concepts or processes.

While it might be argued that these opportunities could be enacted through other pedagogical media, it is the ease of use and the tactile nature of the experience with this app that seems to facilitate these processes more easily, and make them more student directed when the students use the apps. They are also integrated into one device, one that can be moved seamlessly between learning situations. Other students indicated emerging learning and confidence when working on a problem involving money with comments such as: "I'm sort of good at counting money now." Some could not identify specific learning but noted increased confidence. "I still use the same problems and the way I do them, but for some reason I feel more confident doing my maths.”

\section{Discussion and concluding remarks}

Teacher and student responses pointed to the acknowledged potential of the iPad use in manipulating objects dynamically onscreen. The teacher spoke of acting directly with the object, in this case the mathematics, and the students related to tapping and drawing on the screen. Students also commented on using the different digital tools to solve their problems, and so engaging with multi-representations. In this way, the visual and dynamic affordances were acknowledged as part of the emerging theme related to affordances. Some students reflected on examples of new learning through the use of the app, but more clearly the use of screencasting and voice recording had advantages in motivating students and in increasing confidence. As such, the use of the app supports findings regarding visual and dynamic affordances and affective aspects such as motivation, risk-taking and confidence. These related to our emerging themes on engagement and affordances. 


\section{Calder \& Murphy}

Furthermore, the screencasting feature of the app was seen to introduce new representations. It seemed that the simultaneous dynamic visual recording (drawing, manipulating digital tools and writing symbols and words) along with speaking, created a dynamic aural-visual representation. This also resonates with Moyer-Packenham and Westenskow's (2013) simultaneous linking affordance, as the links between different representations influenced the articulation, and by inference, the students' understanding to some extent.

Not only did the teachers and students note that having the multi-representations being used and linked simultaneously was a way to show the thinking processes in solving a word problem, it appeared that, through pausing and editing, the students took time in preparing and perfecting their recordings. They were able to reflect on what had been said and think about what to say next. The teachers spoke of the students creating their own content and language and of having a safe space to do this. There was a creative element to their work in Math Shake. As the students created their own representations and linked these in an individual way when articulating solutions, there was a personal aspect to their screencasts both in the appearance and in the processing of the mathematics. This suggested that some differentiating of the learning had taken place as well, with students following more individual learning trajectories.

The student comments suggested the inter-relationships between the opportunities that the apps afforded and the creating of both individual and group discussion, presentations of solutions, and strategies. They used linked multi-representations to present their thinking. The recordings were then available for the individual students to refer back to as a dynamic aural-visual representation of their own thinking, as well as a representation to share with other students for discussion and with their teachers for assessment and feedback.

\section{Conclusions}

Previous research has suggested that MT can offer affordances that might reshape the learning experience. Through a more immediate and explicit interaction students can manipulate and create dynamic images on the screen to explore mathematical objects. The use of screencasting along with voice recording furthers the multi-representational nature by introducing dynamic aural-visual representations that are created by the students themselves. The process of verbalisation, along with the manipulation of images, drawing and writing in a safe environment that the student controls, would suggest a new learning experience. Further study of this new learning experience is needed to understand how it might be reshaping the learning of mathematics.

Math Shake also offered three of the affordances that Moyer-Packenham and Westenskow (2013) identified. While simultaneous linking resonates with the multi-representation affordance, it also contains elements of instantaneous feedback, where the students' actions and the ensuing onscreen transformations are directly linked. Their notion of creative variation connects to the emerging project themes of personalization and differentiation of the learning, while the affordances of Math Shake constrained the learners' focus on particular virtual learning objects and processes.

Complementing the potential of the affordances of the apps to influence the learning experience, is the pedagogical approach taken by the teacher. The tasks given, and the classroom culture that the teacher develops, are key elements of the learning. The students might have apps available but not necessarily engage with them in ways that optimise the mathematics learning. These aspects were 
implicit in the interview data rather than being commented on directly. However, coupled with observations in the classroom, the importance of the teacher's pedagogical approach seemed clear. For instance, the task of creating a presentation of their solution and strategies opened opportunity to explore and discuss the mathematics through utilising the affordances of Math Shake. The classroom culture including reflecting on processes, exploring in collaborative groupings, and sharing outcomes in a safe learning environment are all conducive to developing mathematical thinking. The seamless engagement with the app, especially in the one-to-one iPad class, was also part of the class culture. The teachers and the students were excited about the learning, and enhanced motivation, cognitive risk taking and confidence were also evident. It appears that it is the assemblage of digital media, learners, mathematics and environmental elements that reshape and hence influence the learning.

The emerging themes of the project and the corresponding framework are being co-constructed with the teacher co-researchers. This process will continue during the second year of the project as the teacher co-researcher group expands into a group of 12 teachers and classes, representing a wide range of year levels, with the teachers having varying levels of experience and expertise in using mobile technologies in their mathematics programmes. This will bring more diverse perspectives as the draft framework evolves through iterations of action, reflection and changing perspectives.

We wish to acknowledge the support of the Teacher and Learning Research Initative.

\section{References}

Attard, C. (2015). Introducing iPads into primary mathematics classrooms: Teachers' experiences and pedagogies. In M. Meletiou-Mavrotheris, K. Mavrou, Katerina, \& E. Paparistodemou (Eds.) Integrating touch-enabled and mobile devices into contemporary mathematics education (pp. 193-213). Hersley PA, USA: IGI Global.

Ainsworth, S. E., Bibby, P. A., and Wood, D. J. (1998). Analyzing the costs and benefits of multirepresentational learning environment, in M. W. van Someren, P. Reimann, H. P. A. Boshuizen, and T. de Jong (Eds.), Learning with Multiple Representations (pp. 120-134). Oxford, U.K.: Elsevier Science.

Arcavi, A., \& Hadas, N. (2000). Computer mediated learning: An exam-ple of an approach. International Journal for Computer for Mathematics Learning, 5, 25-45.

Brown, J. (2005). Affordances of a technology-rich teaching and learning environment. In P. Clarkson, A. Downton, D. Gronn, M. Horne, A. McDonough, R. Pierce, and A. Roche (Eds.). Building connections: theory, research and practice, (Proceedings of the 28th annual conference of the Mathematics Education Research Group of Australasia, Melbourne, (pp.177-184). Sydney: MERGA.

Borba, M. C., \& Villarreal, M. E. (2005). Humans-with-Media and the Reorganization of Mathematical Thinking: Information and Communication Technologies, Modeling, Experimentation and Visualisation, New York, NY: Springer.

Calder, N. S. (2011). Processing mathematics through digital technologies: The primary years. Rotterdam, The Netherlands: Sense.

Calder, N. S. \& Campbell, A. (2016). Using mathematical apps with reluctant learners. Digital Experiences in Mathematics Education. DOI 10.1007/s40751-016-0011-y.

Carr, J. (2012). Does math achievement h'APP'en when iPads and game-based learning are incorporated into fifth-grade mathematics instruction? Journal of Information Technology Education (11) 269-286.

de Freitas, E., \& Sinclair, N. (2014). Mathematics and the body: Material entanglements in the classroom. Cambridge: Cambridge University Press. 


\section{Calder \& Murphy}

Falcade, R., Laborde, C., \& Mariotti, M. A. (2007). Approaching functions: Cabri tools as instruments of semiotic mediation. Educational Studies in Mathematics, 66(3), 317-333.

Fenwick, T., \& Edwards, R. (Eds.)(2012). Researching education through actor network theory. Sussex: Wiley-Blackwell.

Gibson, J. J. (1977). The theory of affordances. In R. Shaw \& J. Bransford (Eds,). Perceiving, acting, and knowing: Toward an ecological psychology (pp. 67-82). Hillsdale, NJ: Lawrence Erlbaum.

Hegedus, S. (2013). Young Children Investigating Advanced Mathematical Concepts With Haptic Technologies: Future Design Perspectives. The Mathematics Educator, 10(1\&2), 87-107.

Hennessy, S. (2014). Bridging between research and practice: Supporting professional development through collaborative studies of classroom teaching with technology. Rotterdam, The Netherlands: Sense.

Melhuish, K., \& Falloon, G. (2010). Looking to the future: M-learning with the iPad. Computers in New Zealand Schools: Learning, Leading. Technology, 22(3), 1-15.

Meyer, B. (2015). iPads in inclusive classrooms: Ecologies of learning. In P. Isaias, J.M. Spector, \& D. Ifenthaler. E-learning systems, environments and approaches theory and implementation. Dordrecht: Springer International Publishing.

Moyer, P. S., Bolyard, J. J., \& Spikell, M. A. (2002). What are virtual manipulatives? Teaching Children Mathematics, 8(6), 372-377.

Moyer-Packenham, P. S., Baker, J., Westenskow, A., Anderson, K., Shumway, J., Rodzon, K., \& Jordan, K, (2013). A Study Comparing Virtual Manipulatives with Other Instructional Treatments in Third- and Fourth-Grade Classrooms. Journal of Education, 193 (2), 25-39.

Moyer-Packenham, P. S., \& Westenskow, A. (2013). Effects of virtual manipulatives on student achievement and mathematics learning. International Journal of Virtual and Personal Learning Environments, 4(3), 35-50.

Philip, T.M. \& Garcia, A. (2014). Schooling mobile phones: Assumptions about proximal benefits, the challenges of shifting meanings, and the politics of teaching. Educational Policy 29 (4), 676-707.

Sacristan, A.I., \& Noss, R. (2008). Computational construction as a means to coordinate representations of infinity. International Journal of Computers for Mathematical Learning. DOI: 10.1007/s10758-0089127-5.

Sandholtz, J.H., Ringstaff, C., \& Dwyer, D.C. (1997). Teaching with technology: Creating a student centred classroom. New York: Teachers' College Press.

Sinclair, N. \& Heyd-Metzuyanim, E. (2014). Learning number with TouchCounts: The role of emotions and the body in mathematical communication. Technology, Knowledge and Learning 19 (1), 81-99. 